Review

\title{
A mechanistic review of Parkin activation
}

\author{
Mehmet Gundogdu ${ }^{\mathrm{a}}$, Roya Tadayon ${ }^{\mathrm{b}}$, Giulia Salzano ${ }^{\mathrm{a}}$, Gary S. Shaw ${ }^{\mathrm{b}}$, Helen Walden ${ }^{\mathrm{a},}$ \\ ${ }^{a}$ Institute of Molecular, Cell and Systems Biology, College of Medical, Veterinary and Life Sciences, University of Glasgow, Glasgow, UK \\ ${ }^{\mathrm{b}}$ Department of Biochemistry, Schulich School of Medicine \& Dentistry, University of Western Ontario, London, ON, Canada
}

\section{A R T I C L E I N F O}

\section{Keywords:}

Parkin

RBR

E3

Autoinhibition

Ubiquitylation

\begin{abstract}
A B S T R A C T
Parkin and phosphatase and tensin homolog (PTEN)-induced kinase 1 (PINK1) constitute a feed-forward signalling pathway that mediates autophagic removal of damaged mitochondria (mitophagy). With over $130 \mathrm{mu}$ tations identified to date in over 1000 patients with early onset parkinsonism, Parkin is considered a hot spot of signalling pathways involved in PD aetiology. Parkin is an E3 ligase and how its activity is regulated has been extensively studied: inter-domain interactions exert a tight inhibition on Parkin activity; binding to phosphoubiquitin relieves this auto-inhibition; and phosphorylation of Parkin shifts the equilibrium towards maximal Parkin activation. This review focusses on recent, structural findings on the regulation of Parkin activity. What follows is a mechanistic introduction to the family of E3 ligases that includes Parkin, followed by a brief description of structural elements unique to Parkin that lock the enzyme in an autoinhibited state, contrasted with emerging models that have shed light on possible mechanisms of Parkin activation.
\end{abstract}

\section{Introduction}

Parkinson's disease (PD) was first characterised in 1817 based on a triad of motor symptoms: tremor, rigidity and bradykinesia. Today, PD constitutes the most common movement disorder, affecting $0.1 \%$ of the global population, with the prevalence increasing to $4 \%$ with advancing age $[1,2]$. Motor symptoms in PD stem from loss of dopaminergic neurons in the substantia nigra pars compacta, yet despite this understanding of the pathophysiology there is a lack of available biomarkers for clinical use. Thus, accurate diagnosis of patients often occurs only once more than $30 \%$ of all nerve cells in the pars compacta have been lost, after which point the motor symptoms typically appear [3]. There is no cure for $\mathrm{PD}$, with available treatments limited to symptomatic management options that may only provide benefit briefly, before losing their efficacy [4].

A breakthrough in understanding of PD molecular mechanisms came with an earlier observation that exposure to a mitochondrial toxin can cause a rapid-onset PD, implicating mitochondrial dysfunction in PD pathology [5]. Subsequently, numerous studies have associated a combination of genetic mutations on 11 genes with heritable forms of PD [6]. However, mutations at so-called "PARK" loci are estimated to underly the pathogenesis of $<5 \%$ of all PD cases. Remarkably, the evidence on genetic predispositions and environmental factors both highlighted that the aetiology of PD involves proteins implicated in maintenance of mitochondrial function or degradation of dysfunctional mitochondria [7].

Parkin (PARK2) and phosphatase and tensin homolog (PTEN)induced kinase 1 (PINK1; PARK6) may harbour mutations that cause an autosomal recessive form of early onset $\mathrm{PD}$, where the age at onset can be below 20 [8-10]. With over 130 distinct mutations identified in over 1000 patients, and observed in $20-71 \%$ of all cases, biallelic Parkin mutations are the most common cause of early onset PD [11-13]. Thus, Parkin has been under major investigation with the aim of identifying cell signalling pathways involved in PD aetiology.

Parkin is a cytosolic member of the Really Interesting New Gene (RING)-in-between-RING (IBR)-RING (RBR) ubiquitin (UB) ligase family with an N-terminal UB-like (UBL) domain, while PINK1 is a protein kinase with mitochondrial targeting sequence $[10,11]$. The protein ubiquitylation cascade and Parkin topology are discussed in greater detail in Sections 2 and 3, respectively. The functions of these proteins are linked in a feed-forward signalling pathway that mediates autophagic removal of damaged mitochondria (mitophagy). In this pathway, depolarisation of mitochondria prevents constitutive, presenilin-associated rhomboidlike protein (PARL)-protease-dependent degradation of PINK1, allowing PINK1 to accumulate specifically on dysfunctional mitochondria $[14,15]$. PINK1 then phosphorylates UB chains linked to outer mitochondrial membrane (OMM) proteins [16-18]. This Ser65phosphorylated UB (pUB) recruits Parkin to mitochondria, where

\footnotetext{
* Corresponding author.

E-mail address: Helen.Walden@glasgow.ac.uk (H. Walden).
} 
PINK1 can subsequently phosphorylate Parkin's UBL domain at Ser65, at a site homologous with the PINK1 UB phosphorylation site [19-21]. While cytosolic Parkin displays attenuated activity, once bound to pUB and phosphorylated by PINK1, the auto-inhibition of Parkin is fully relieved [22,23]. Upon activation, Parkin modifies various OMM proteins, predominantly with K11- and K63-linked UB chains [24]. Parkindependent ubiquitylation of OMM proteins generates more substrate for PINK1, thus increasing the pool of pUB on damaged mitochondria and closing the feed-forward loop. Ultimately, (p)UB-chains on mitochondria recruit autophagy receptors and promote autophagosome assembly at the damaged mitochondria [25-27].

PINK1-Parkin signalling is well understood and has recently been reviewed in greater detail elsewhere [28,29]. Aiming to avoid redundancy with recent, extensive reviews on Parkin signalling, the focus will be on recent, structural findings on the regulation of Parkin activity. What follows is a short, mechanistic introduction to the RBR type E3 ligase family that includes Parkin (Section 2), followed by a brief review of the structural elements unique to Parkin that lock Parkin in an autoinhibited state (Section 3), contrasted with emerging models that have shed light on possible mechanisms of Parkin activation (Section 4).

\section{Hybrid mechanism of ubiquitin transfer}

Ubiquitylation is a post-translational modification involving the concerted actions of UB-activating (E1), UB-conjugating (E2) and UBligating (E3) enzymes. The human genome harbours two E1, approximately $40 \mathrm{E} 2$ and over 600 validated E3 enzymes [30]. The E1 activates the $\mathrm{C}$-terminus of $\mathrm{UB}$, triggering transfer of $\mathrm{UB}$ onto the $\mathrm{E} 2$ catalytic cysteine [31,32]. Once "loaded" with UB, the E2 UB conjugate (where $\sim$ denotes thioester linkage) functions as a UB transporter [33]. Transfer of UB onto substrates, however, requires E2 UB conjugates to pair with E3s, which ensure substrate selection and catalyse transfer of UB onto the substrate lysine. Substrate proteins can be modified with a single UB on a single or multiple lysine residues, termed mono, or multimono ubiquitylation. Moreover, the UB moiety on the substrate protein can stimulate the formation of UB chains through one of its seven internal lysine residues, termed poly-ubiquitylation [34]. Protein ubiquitylation can thereby generate myriad structurally distinct signals that trigger various cell signalling events ranging from proteasomal degradation [35] and DNA repair [36-39] through to lysosomal targeting [40].

E3 ligases play a key role in directing substrate specificity and triggering transfer of UB from E2 UB conjugates onto specific substrate lysine residues. The large number of $\mathrm{E} 3$ ligases can be categorized into three groups on the basis of the reaction mechanism they employ: RING type, HECT (Homologous to E6-AP C-Terminus) type, and RBR type [41]. The RING type E3 ligases act as a scaffold, binding to E2 $\sim \mathrm{UB}$ and catalysing direct transfer of UB from E2 $\sim \mathrm{UB}$ to a lysine residue in the substrate [42-44]. HECT type E3 ligases, on the other hand, follow a different mechanism, where the N-terminal lobe of the HECT domain recruits the $\mathrm{E} 2 \sim \mathrm{UB}$ conjugate and mediates transfer of the UB cargo onto the catalytic cysteine residue of HECT C-terminal lobe, thereby forming an E3 UB thioester linkage prior to substate ubiquitylation [41,45-47]. The RBR E3 ligase family, which Parkin belongs to, were originally defined based on their three $\mathrm{Zn}^{2+}$-coordinating RBR subdomains: (1) RING1 domain, which shares homology with canonical RING domains and engages the E2 UB; (2) IBR domain, which regulates activity by binding allosteric UB molecules; and (3) RING2, which contains a catalytic cysteine to form an E3 UB [48,49]. RBR type E3 ligases display a RING/HECT hybrid mechanism that mediates substrate ubiquitylation, whereby RBR RING1 interacts with the E2 $\sim$ UB conjugate in a RING E3-like fashion, but with a requirement for the donor UB to be transferred onto the RBR RING2 catalytic cysteine prior to substrate ubiquitylation in a manner similar to HECT-type E3s [50]. Of note, IBR and RING2 domains display a conserved fold (2.6 A RMSD between 40 overlapping $C_{a}$ atoms between IBR and RING2) [51] that is distinct from that of canonical RING domains (8.5 ̊ RMSD between 32 overlapping $\mathrm{C}_{\mathrm{a}}$ atoms between Parkin RING2 and RING finger protein 4 [RNF4] RING domain [PDB: 4AP4]) [25,52]. Reflecting this, alternative nomenclature highlighting the function of these domains has been proposed, with the IBR and RING2 domains termed the benign-catalytic (BRcat) and required-for-catalysis (Rcat) domains, respectively [53].

Although a common feature of all E3 ligases is the ability to interact with an $\mathrm{E} 2 \sim \mathrm{UB}$ conjugate, $\mathrm{E} 2 \sim \mathrm{UB}$ conjugates are highly dynamic in solution and can adopt an array of conformations. Often, the conformation of the E2 UB conjugate is influenced by the presence of an E3 ligase [54-56]. For instance, whilst in solution UBE2D2 UB forms a highly dynamic and extended "open" conformation with only transient interaction surfaces observed between UB and E2 [55,57]. Alternatively, UBE2L3 UB, which discharges UB preferentially to cysteine [50], prefer a "closed" arrangement [58] where the hydrophobic patch formed around UB Ile44 interacts directly with the E2 crossover helix, first identified for yeast Ubc1 UB [59]. In contrast, E2 UB conjugates in the presence of a RING-type E3 enzymes such as baculoviral IAP repeat containing protein 7 (BIRC7) or RNF4 are typically stabilized in the "closed" conformation $[25,57,60]$, while UBE2L3 and UBE2D conjugates switch to an "open" state in the presence of the RBR E3 ligases Heme-oxidized IRP2 UB ligase 1 (HOIL-1)-interacting protein (HOIP), Human Homolog of Ariadne (HHARI) and Parkin.

Induction of a closed E2 $\sim$ UB conformation is a key feature of the RING E3 ubiquitylation mechanism. In the RING-type E3:E2 UB complex, the contact surface between the E2 and RING E3 proteins is approximately $15 \AA$ away from the $\mathrm{E} 2$ active site and includes $\mathrm{Zn}^{2+}$. coordinating loops and the RING central helix (Fig. 1A). A conserved residue at the C-terminal end of the RING core, typically arginine, lysine, or asparagine, interacts with the E2 carbonyl backbone as well as the UB tail, serving as a so-called "linchpin" in RING E3:E2 UB complexes. The linchpin residue contributes to stabilisation of $\mathrm{E} 2 \sim \mathrm{UB}$ in the "closed" conformation, allowing the substrate lysine to initiate a nucleophilic attack on the E2 $\sim$ UB thioester and thus receive the UB [50,57,61].

RING1 domains in RBR E3 ligase family members such as Parkin, HOIP, HHARI are structurally similar to canonical RING domains and encompass a classical cross-brace arrangement and $\mathrm{Zn}^{2+}$-coordination topology [44,62]. Nevertheless, RBR RING1 domains lack the linchpin mechanism required to stabilise the "closed" conformation of E2 $\sim$ UB [63]. Moreover, the crystal structure of HHARI in complex with UBE2L3 $\sim$ UB (PDB 5UDH) [63] reveals that the E3:E2 interface involves a loop on RING1 that is two residues longer than its canonical RING E3 counterparts, thereby preventing formation of the "closed" E2 $\sim$ UB conformation by obstruction of the donor UB [63]. The crystal structure of HOIP:UBE2D2 UB further supports this stabilisation of the E2 UB in the "open" state $[64,65]$. While there is no structure of Parkin in complex with a E2 UB conjugate, the aforementioned loop extension is conserved across all RBR E3 ligases [53], including Parkin, suggesting that promotion of an "open" E2 $\sim$ UB conformation may be a common feature of all RBR E3 ligases (Fig. 1B) [58,60]. It is generally understood that by promoting "open" E2 UB states, RBR E3s prevent direct UB transfer onto substrate lysine residues, thereby forcing the reaction mechanism to go through the Rcat catalytic cysteine (Fig. 1C) [64]. In line with this, substitution of HHARI RBR RING1 with a canonical RING imparts the ability to promote the "closed" E2 UB conformation, conferring the synthetic RING/RBR hybrid the ability to catalyse direct substate ubiquitylation, without the need to utilise Rcat catalytic cysteine [64].

\section{Regulation of Parkin by auto-inhibition and allosteric activation}

Parkin-mediated ubiquitylation can only take place once Parkin RING1 engages E2 UB and the donor UB is transferred from the E2 catalytic cysteine to Cys431 on Parkin Rcat, termed transthiolation 
A

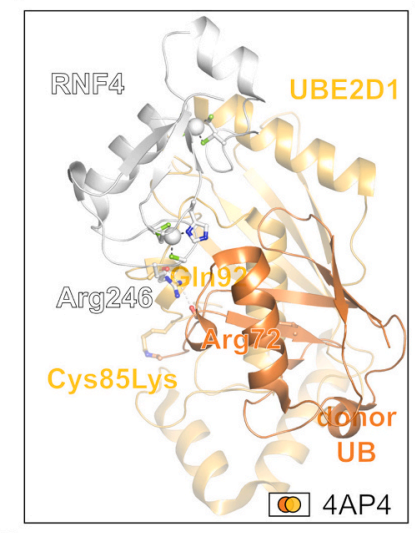

C

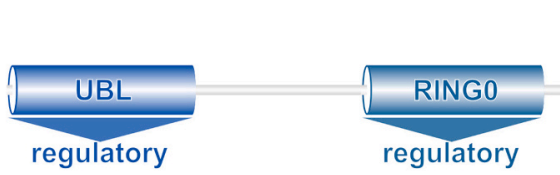

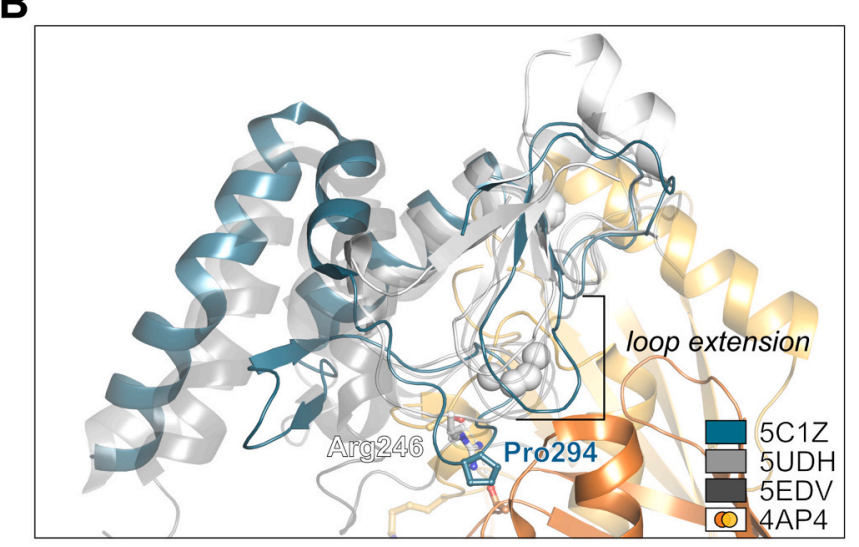

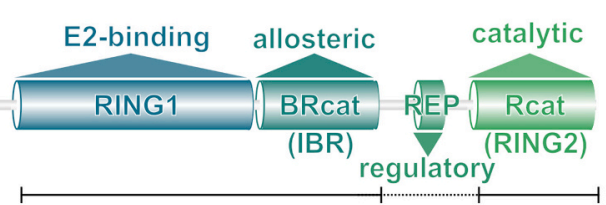

canonical RBR module

Fig. 1. Differing mechanism of E2 $\sim \mathrm{UB}$ recruitment by canonical RING E3s and RBR RING1.

(A) Ribbon representation of RNF4 RING domain bound to UBE2D1 UB (PDB: 4AP4 [25]). $\mathrm{Zn}^{2+}$ ions are shown as white spheres and residues involved in their coordination are shown as sticks. Side chain of RNF4 linchpin Arg246 and backbone carbonyls of UBE2D2 Gln92 and UB Arg72 are also shown as sticks, with hydrogen bonding interaction between them depicted as white dashed lines. (B) Same as in (A), but with RING1 domains of Parkin (PDB: 5C1Z [73]), HHARI (PDB: 5UDH [63]) and HOIP (PDB: 5EDV [64]) superimposed on RNF4 RING domain. Parkin Pro294, corresponding to RNF4 linchpin Arg246 is shown as sticks. RING1 loop extension relative to RNF4 RING is also demarcated. (C) Parkin domain organisation diagram drawn to scale, illustrating Parkin UBL, RING0, RING1, BRcat (IBR), REP and catalytic Rcat (RING2) domains coloured from blue to green, respectively.
(Section 2) [67]. It has been extensively shown, however, that Parkin exists in a compact, autoinhibited conformation, where the UBL domain exerts an inhibitory effect on both E2 binding and transthiolation
$[68,69]$. Auto-inhibition of Parkin transthiolation, with its allosteric release is discussed further in Section 4 in the context of prominent Parkin activation models. The focus of this section will be on how the
A

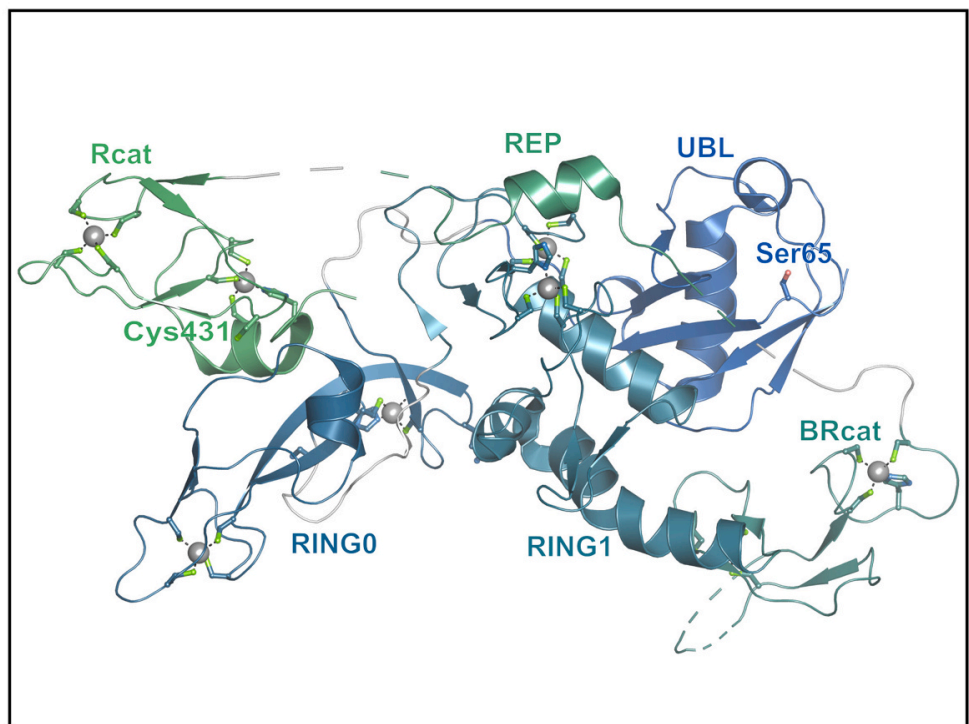

B

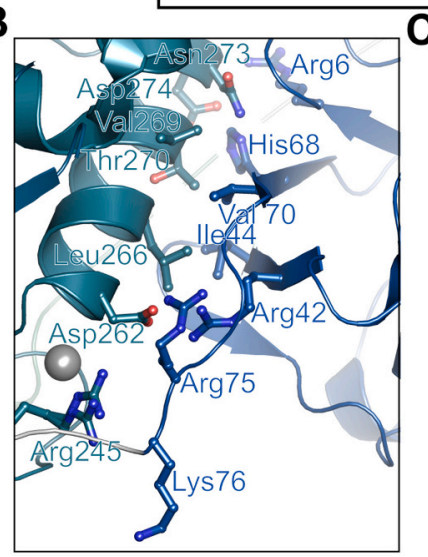

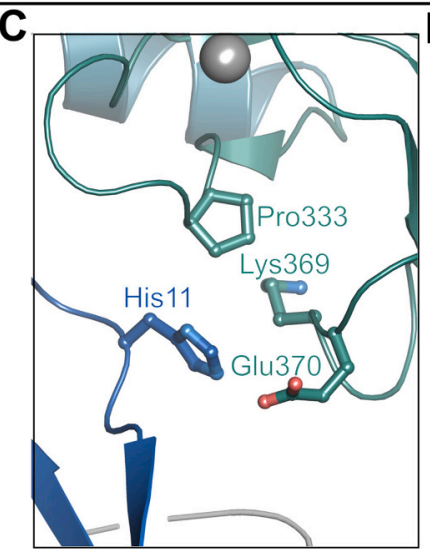

Fig. 2. Structure of Parkin in the inactive state.

(A) Ribbon representation of inactive Parkin (PDB: 5C1Z [93]) showing UBL, RING0, RING1, BRcat, REP and Rcat domains coloured from blue to green, respectively. $\mathrm{Zn}^{2+}$ ions are shown as white spheres and residues involved in their coordination are shown as sticks. UBL Ser65 and catalytic Rcat Cys431 are also shown as sticks. (B) Close-up of interface between UBL and RING1 domains. (C) Close-up of interface between UBL and BRcat domains. (D) Close-up of interface between REP and RING1 domains, with the RING1 surface shown and coloured to indicate hydrophobic, negatively charged and positively charged regions yellow, red and blue, respectively. 
Parkin UBL domain and the linker connecting the BRcat and Rcat domains, termed as the repressor element (REP), occlude the E2-binding site on Parkin RING1 (Fig. 2A), and how dual-phosphorylation by PINK1 can relieve this autoinhibition [62,70,71].

\subsection{Inhibition of $E 2 \sim U B$ binding}

Several crystal structures have shown that the compact autoinhibited structure is primarily driven by large interfaces between the UBL domain and the rest of the structure [72,73]. The most extensive interface is defined by several UBL-RING1 contacts and includes hydrophobic interactions between UBL Ile44 and Val70 and RING1 Leu266, Val269 and Thr270 (Fig. 2B). Also, UBL Arg42 and RING1 Asp262 form a salt bridge, while UBL Arg6 and His68 tether RING1 Asn273 and Asp274. The N-terminus of the UBL domain is further stabilized by UBL Arg75 and Lys76 interacting with RING1 Arg245 (Fig. 2B). A further interface is formed between the UBL and BRcat domains, with UBL His11 interacting with BRcat Pro333, Lys369 and Glu370 (Fig. 2C). The compact packing of the Parkin UBL domain onto the RBR module also explains the earlier observation that $\mathrm{N}$-terminal tags on Parkin may relieve autoinhibition [74-76]. N-terminally tagged Parkin displays in vitro activity due to interference with these inhibitory intramolecular interactions, with cleavage of the tag restoring autoinhibition [77]. In line with this, while a fragment of Parkin composed of the RING0 and RBR domains (hereafter RORBR) displays higher residual activity than wild-type Parkin, titration of UBL restores autoinhibition of this fragment [73]. Point mutations that disrupt these inhibitory interactions activate Parkin both in vitro and in cells [72,78].
Moreover, presence of pathological PD mutations in the UBL domain, which abolish the autoinhibited conformation of Parkin, further confirm the importance of compact UBL-R0RBR packing for auto-inhibition of Parkin activity.

The other auto-inhibitory element blocking E2 recruitment on Parkin RING1 is the REP region. The REP helix is tethered between the two $\mathrm{Zn}^{2+}$-coordination centres in Parkin RING1, with Tyr391 and Trp403 on either end of the REP helix protruding into hydrophobic patches found on RING1 surface and cementing this interaction (Fig. 2D). Mutation of either of these hydrophobic REP anchors disrupts the REP-RING1 interface and leads to Parkin activation [72].

\subsection{Allosteric release by pUB-binding}

As described above, Parkin activity is tightly regulated by interdomain interactions. Recruitment of Parkin on pUB or direct phosphorylation of Parkin at the UBL domain can relieve the auto-inhibition of $\mathrm{E} 2 \sim \mathrm{UB}$ binding and transthiolation independently $[18,20,21,23,79]$. Maximal E3 ligase activity, however, requires synergistic effect of both events. [78,80] With over 10 crystal structures investigating different aspects of Parkin inhibition in the Protein Data Bank (PDB), the allosteric mechanisms behind Parkin activation are being revealed [44,62,68,69,72,73,81-83].

Central to the allostery behind the release of Parkin activity is the RING1 domain. RING1 is comprised of two lobes: the canonical crossbrace fold consisting of one central helix flanked by two zinccoordinating $\beta$-hairpin loops; and the C-terminal extension unique to RBRs that fold into one short and one longer hairpin and kinked $\alpha$-helix

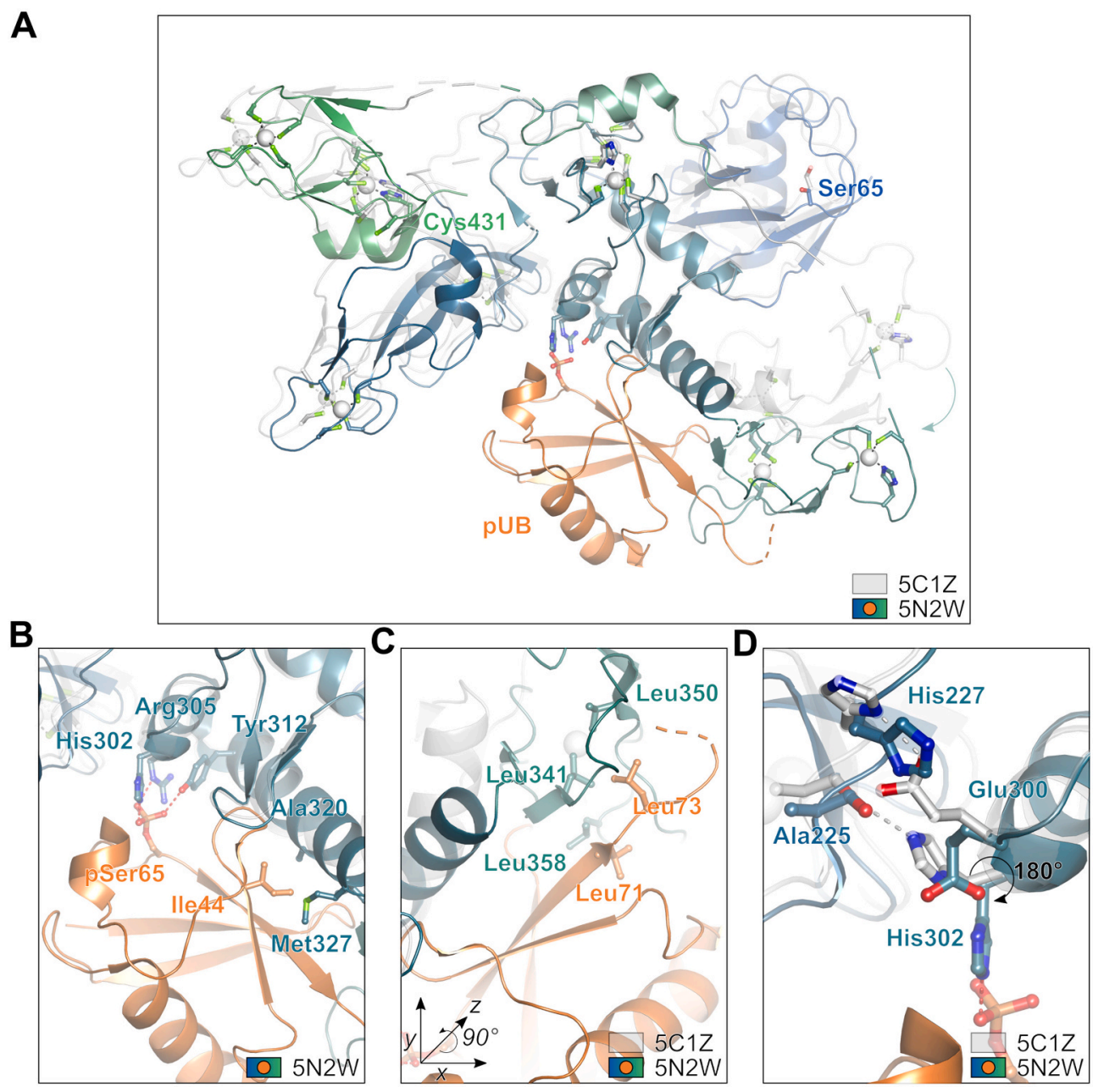

Fig. 3. Allostery behind Parkin activation by pUB-binding.

(A) Ribbon representation of Parkin:pUB complex (PDB: 5N2W [69]) coloured and viewed as in Fig. 2A, with pUB also shown as ribbons and coloured orange. Superimposition of inactive Parkin (PDB: 5C1Z [73]) is shown as white ribbons for comparison between inactive and pUB-bound states. $\mathrm{Zn}^{2+}$ ions are shown as white spheres and residues involved in their coordination are shown as sticks. UBL Ser65, Rcat catalytic Cys431, pUB pSer65, and RING1 residues involved in phosphate-coordination are also shown as sticks. (B) Close-up of the pUB coordination by Parkin RING1 helical extension region. (C) Close-up pUB C-terminal tail and Parkin BRcat interaction. Spatial relation between panels A and C is illustrated. (D) Close-up of the network of hydrogen bonds between Parkin RINGO and Rcat domains that is disrupted by pUB binding. Colouring of Parkin domains and pUB is as described in Fig. 2A. Spatial relations of panels $\mathrm{A}, \mathrm{B}$ and $\mathrm{C}$ relative to Fig. $2 \mathrm{~A}$ is illustrated where different. 
(Fig. 1B) (hereafter RING1 helical extension). The former lobe mediates E2-binding, while the latter serves as an allosteric UB-binding site (Fig. 3A).

During pUB binding, His302, Arg305 and Tyr321 side chains at the $\mathrm{N}$-terminus of the RING1 helical extension, near the RING0-RING1 interface, tether phospho-Ser65 residue from pUB, while at the C-terminus, nonpolar Ala320, Val324 and Met327 provide an interaction surface for pUB's hydrophobic Ile44 patch (Fig. 3B). One of the RING1 $\beta$-hairpins interdigitates the two helices near the RING1 helical extension, buttressing the interaction between the pUB Ile44 loop and the RING1 helical extension region. In this arrangement, the UB tail threads into the central hydrophobic grove in the Parkin BRcat domain, where the side chains of UB Leu71 and Leu73 insert between that of BRcat Leu358, Leu341 and Val350 (Fig. 3C). This interaction between the pUB tail and BRcat straightens the long helix in the RING helical extension and swings the BRcat domain approximately $20^{\circ}$ away from RING1, thereby creating a deep cleft between RING1 and BRcat (Fig. 3A and C) $[69,81,82]$. The mobility of the BRcat domain had previously been noted from NMR dynamics data and comparison of multiple crystal structures [73]. Remarkably, opening of this cleft between RING1 and BRcat exposes a cryptic UB-binding region that is predicted to interact with the donor UB in the E2 $\sim$ UB conjugate. This is discussed in greater detail in Section 3.3.

RING1 also serves as a conduit for hydrogen-bonding interactions that mediate crosstalk between pUB binding and UBL phosphorylation. When Parkin is autoinhibited, the RING1 His302 side chain hydrogen bonds with the RING0 Ala225 backbone carbonyl, with the adjacent RING1 Glu300 tethering RING0 His227. Binding of pUB causes the His302 side chain to swing by $\sim 180^{\circ}$, thereby disrupting this hydrogenbonding network (Fig. 3D). This weakening of the RING0-RING1 interface has a knock-on effect on RING1-UBL interface at the opposite side, increasing the likelihood of UBL phosphorylation. This is illustrated by the fact that while UBL can bind in trans to a fragment of Parkin composed of RING0-RBR with sub-micromolar affinity, no such interaction can be detected when Parkin is bound to pUB [73]. Not surprisingly, therefore, efficiency of Parkin UBL phosphorylation by PINK1 is markedly higher when Parkin is bound to pUB [82]. Reciprocally, introduction of a negative charge on Ser65 of Parkin UBL can disrupt the network of hydrogen bonds tethering the RING0-RING1 interface, increasing availability of the pUB binding site [73]. In line with this observation, Parkin displays sub-micromolar affinity for pUB, but when UBL domain is phosphorylated, this affinity is approximately 20 -fold higher $[69,81,82]$.

\section{3. $E 2 \sim U B$ binding}

The two inhibitory interactions that block E2 recruitment by Parkin RING1, involving the UBL and REP, appear intact in a complex of Parkin bound to pUB, its allosteric activator (Fig. 3A). Nevertheless, comparison of the Parkin:pUB and phospho-Parkin (pParkin) mimic ParkinSer65Asp:pUB complexes reveal how introduction of a negative charge on UBL Ser65 abolishes a key interaction that tether UBL and REP together and to RING1 [69]. The 17 amino acid linker connecting REP to the BRcat is partly ordered in the Parkin:pUB complex and can be seen threading between UBL and RING1. In this arrangement, the REP-BRcat linker residue Tyr391 interacts with Tyr267 on the central RING1 helix, while the nearby linker residue Gln389 is within van der Waals radius of Ser65 on UBL. Introduction of negative charge on UBL Ser65 disrupts this Gln389-Ser65 interaction, pushing the REP-BRcat linker out and destabilising the UBL-REP-RING1 interface (Fig. 4A) [69]. In line with this, while autoinhibited Parkin does not display detectable binding affinity towards E2s, pParkin:pUB binds UBE2L3 with low-micromolar affinity $[69,73]$.

A recent study reported a crystal structure of phosphorylated Bactrocera dorsalis Parkin bound to pUB and in complex with UBE2L3 (hereafter pBdParkin:pUB:UBE2L3) [83], and RORBR Parkin bound to pUB and a UBE2L3 UB conjugate [65], confirming that E2 recruitment by activated Parkin follows what is observed in conventional RING E3E2 interactions (Section 2) [84,85]. Namely, negatively charged residues on the Parkin RING1 central helix and the first $\mathrm{Zn}^{2+}$-coordinating loops tether Arg5 and Arg7 within UBE2L3 N-terminal helix, while hydrophobic residues on the Parkin RING1 central helix and the second $\mathrm{Zn}^{2+}$-coordinating loop interact with UBE2L3 Phe63 and Pro97 on loops 4 and 7, respectively (Fig. 4B). Both structures show that, when in complex with activated Parkin, the UBE2L3 active site points towards the deep UB-binding cleft between RING1 and BRcat (see Section 3.2). The structure of R0RBR:pUB:UBE2L3 UB shows that Parkin stabilises the UB moiety in the "open" state, with the donor UB nestled in the RING1-BRcat cleft [65]. This cleft was first identified in a high resolution crystal structure of human Parkin complexed with pUB that showed this region between Parkin RING1 and BRcat is occupied by a UBL domain of a neighbouring, symmetry related Parkin molecule [69]. Secondly, superposition of the RING1 domain of a HOIP:UBE2D2 UB complex onto Parkin RING1 positions the donor UB in this cleft (Fig. 4C) [86]. In this cryptic UB-binding pocket, Arg275 of the Parkin RING1 central helix and Tyr318 and Glu321 of the RING1 helical extension are proposed to coordinate the donor UB. Detailed mutagenesis and in vitro interaction studies have shown that pParkin:pUB with the cryptic UBbinding site mutation Glu321Ala displays wild-type level binding to
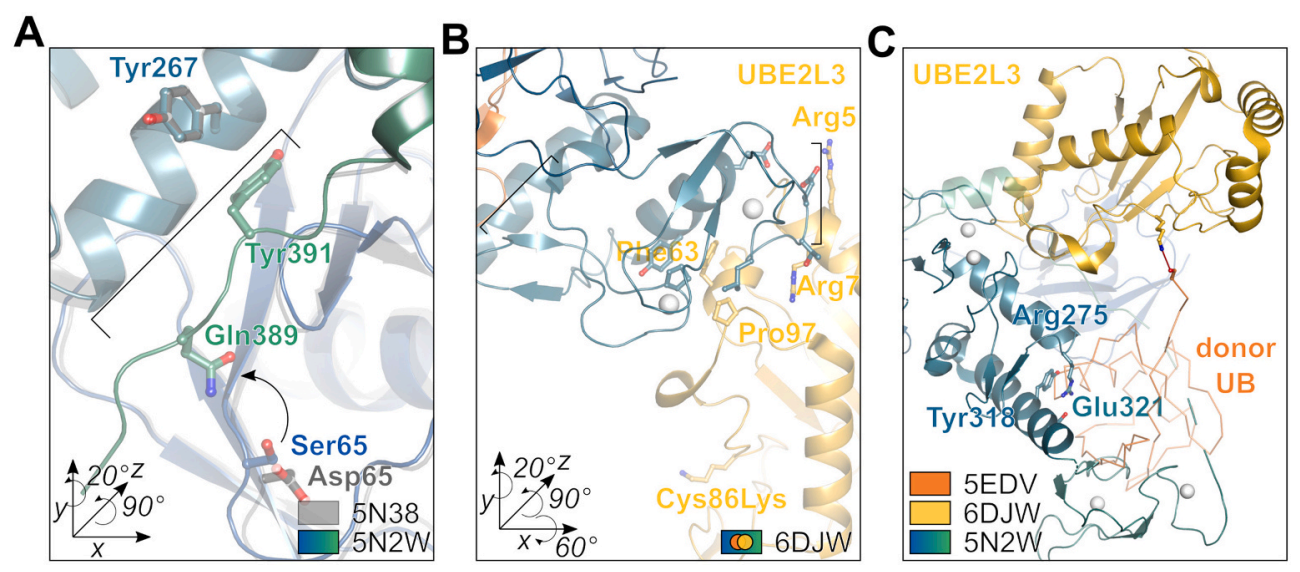

Fig. 4. Disruption of Parkin auto-inhibition and $\mathrm{E} 2 \sim \mathrm{UB}$ recruitment.

(A) Close-up of RING1-UBL interface on Parkin:pUB complex (PDB: 5N2W [69]) overlaid with that of Parkin-Ser65Asp:pUB (PDB: 5 N38 [69]), highlighting the destabilisation caused by negative charge on UBL residue 65. (B) Close-up of RING1-UBE2L3 interaction seen in $\mathrm{pBdParkin}$ :pUB:UBE2L3 complex (PDB: 6DJW [83]). (C) Ribbon/line representation of Parkin bound to UBE2L3 UB, showing donor UB nestled in the cryptic donor UB-binding region. This state is modelled by superimposing RING1 of inactive Parkin (PDB: 5C1Z [73]), pBdParkin:pUB:UBE2L3 complex (PDB: 6DJW [83]) and HOIP:UBE2D2 UB complex (PDB: 5EDV [64]). For clarity, only inactive Parkin is shown, with UBL and REP rendered semitransparent, with the UBE2L3 and the donor UB shown as yellow ribbons and orange lines, respectively. Spatial relations of panels B, C and D relative to Fig. $2 \mathrm{~A}$ is illustrated where different. 
UBE2L3 alone, while binding to UBE2L3 UB conjugate by a 20 -fold reduced affinity [69]. This corroborates the function of the deep RING1-BRcat cleft as the cryptic UB-binding site. Indeed, mutation of UB-binding cleft residues hampers E3-ligase activity [69].

\section{Complete Parkin activation: cis- and trans-activation models}

Probably the most enigmatic aspect of Parkin activation has been the observation that even when Parkin is bound to pUB, the catalytic Cys431 in Rcat is poorly accessible at RING0-Rcat interface, and remains $\sim 50 \AA$ away from the E2 binding site on RING1 (Fig. 3A) [44,62,72,73]. Over the last decade two models attempting to explain the mechanism underlying UB-transfer by Parkin have gained attraction and these are the so-called trans- and cis-activation models.

\subsection{Priming for UB-transthiolation}

The trans-activation model proposes that multiple Parkin molecules cooperate to overcome the distance between Rcat Cys431 and E2 UB thioester and achieve transthiolation. There is no structural model showing directly how Parkin may achieve trans-activation, but a domain-swap model exists for the evolutionarily related RBR E3 ligase HOIP, suggesting that trans-activation may exist in other RBR E3 ligases [64]. In the HOIP:UBE2D2 UB model, HOIP can be seen forming a homodimer along its RING-BRcat linker region, which corresponds to REP in Parkin, and coordinating the E2 moiety of UBED2 UB via the RING1 domain of one HOIP molecule, while also engaging the UB moiety via the Rcat domain of the other HOIP molecule [64].
Several in vivo and in vitro observations also suggest that this transactivation model is relevant to Parkin. Firstly, overexpression of either catalytically dead Parkin Cys431Ala or E2 UB-binding-deficient Parkin Arg275Trp in HeLa cells, which have low levels of endogenous Parkin, fails to induce mitophagy, yet when the two are co-expressed, this defect is rescued [87,88]. Similarly, pParkin with a cryptic E2 UB binding site mutation, Glu321Ala, displays attenuated binding to UBE2L3 UB and fails to ubiquitylate Miro1 in vitro, with titration of a covalently linked Parkin-pUB complex rescuing this Miro1 ubiquitylation defect [69]. This implies that while the mutant Parkin fails to bind to the donor UB, it can still interact with the E2, while the wild-type pUB-Parkin can bind the donor UB, mediating formation of an active complex that involves 2 Parkin assemblies around one E2 $\sim$ UB conjugate. On the contrary, however, a recent report has shown that titration of E2 UB-binding deficient Rattus norvegicus Parkin Ala240Arg (Thr240 in human Parkin) on a catalytically dead Parkin does not lead to Parkin auto-modification in vitro [83]. It is possible, therefore, that cooperation between multiple Parkin molecules is facilitated by presence of a physiological substrate and does not happen in the minimalist set up of an in vitro self-modification assay.

Alternatively, the cis-activation model implies that following pUB binding and phosphorylation of the UBL domain, Parkin can unravel, allowing the Rcat domain to traverse across the central RING1 domain to meet the E2 UB conjugate. Several recent reports produced crystallographic and NMR/MD simulation-driven models that illustrate the mechanism behind this cis-activation (Fig. 5A) [65,68,83,89]. In this model, once phosphorylated, the UBL domain is released from its RING1 site [90] and rebinds through RING0 Lys161, Arg163 and Lys211 on the

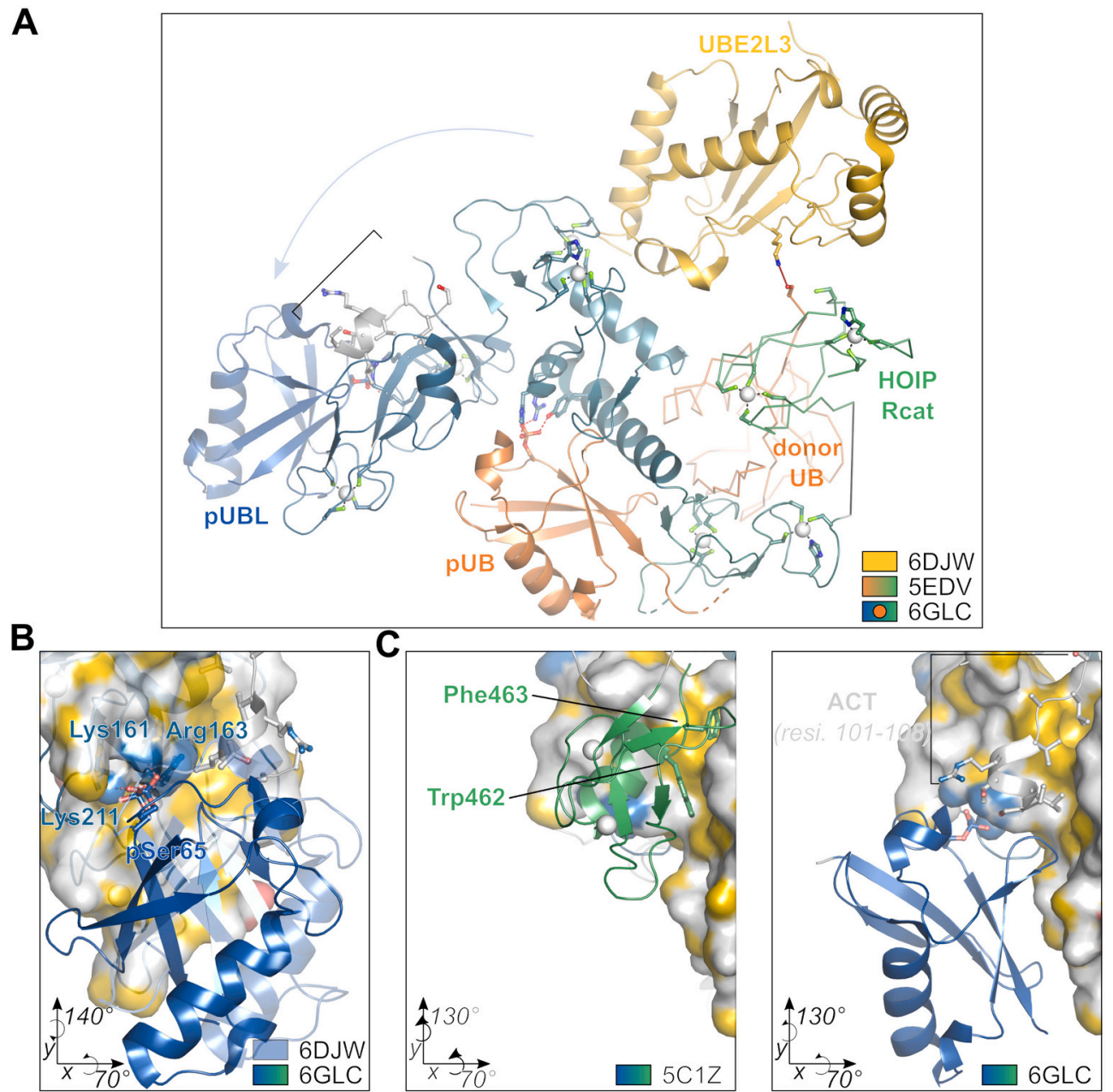

Fig. 5. Parkin in its fully active state and modelled as poised to receive donor UB.

(A) Ribbon representation of pParkin ${ }_{\triangle \mathrm{REP}} \triangle \mathrm{R}$ cat:pUB complex (PDB: 6GLC [68]) coloured and viewed as in Fig. 1A. Fully activated state is modelled by superimposing RING1 of fully-active pParkin $\triangle$ REP $\triangle$ Rcat (PDB: 6GLC [68]), pBdParkin:pUB:UBE2L3 complex (PDB: 6DJW [83]) and HOIP:UBE2D2 UB complex (PDB: 5EDV [64]). For clarity, only fully active pParkin ${ }_{\triangle R E P \triangle R c a t}: \mathrm{PUB}$ is shown, with the UBE2L3 and the donor UB shown as yellow ribbons and orange lines, respectively. HOIP Rcat is also shown as lines, with the catalytic cysteine poised to receive the donor UB shown as sticks, and E2 UB thioester represented as solid red line. (B) Close-up of pUBL-RING0 interaction as seen in pParkin ${ }_{\triangle \mathrm{REP} \triangle \mathrm{Rcat}}$ :PUB (PDB: 6GLC [68]) and $\mathrm{p} B d$ Parkin:pUB:UBE2L3 complex (PDB: 6DJW [83]), where the two Parkin molecules are superimposed over RING0 domain, pParkin is coloured as in Fig. $1 \mathrm{~A}$ and $\mathrm{p} B d$ Parkin is shown as blue ribbons and rendered semi-transparent. RING0 surface is shown and coloured to indicate hydrophobic, negatively charged and positively charged regions yellow, red and blue, respectively. (C) Same as in (B), but highlighting the overlapping RINGO-Rcat (left; PDB: 5C1Z [93]) and RING0-pUB/ACT (right; PDB: 6GLC [68]) interfaces. 
RING0 domain, with additional contacts mediated by the UBL hydrophobic patch around Ile44 and one of the RING0 $\beta$-hairpin loops (Fig. 5B) $[68,83]$. As the Rcat and pUBL binding sites on RING0 display an overlap (Fig. 5C), this ionic pUBL-RING0 interaction is able to displace Rcat, which is tethered on RING0 predominantly by weak hydrophobic interactions $[68,83]$. Once Rcat is dislodged from RING0, the hydrophobic groove on RING0 becomes accessible.

The UBL is linked to RING0 via a 66-residue linker in human Parkin, which is disordered in autoinhibited Parkin structures [72]. Remarkably, in the pParkin ${ }_{\triangle R E P} \triangle \mathrm{Rcat}: \mathrm{PUB}$ complex, a section of the UBL-RING0 linker, from Gln100 through to Ser108, is ordered on the RING0 hydrophobic groove. Critically, linker residues Leu102, Val105 and Leu107 cover the hydrophobic groove on RING0 that is exposed by departure of Rcat, while linker residue Arg104 make a salt bridge with pUBL Asp60, thus stabilising Parkin in the activated state by preventing Rcat from rebinding to RING0 and competing with the pUBL:RING0 interaction (Fig. 5A and C) [68]. Interestingly, while the length of this linker is conserved in most species of Parkin, with the exception of nematodes, sequence conservation is seen only in the middle section corresponding to Ser101 through to Leu123 in the human sequence and exists only among vertebrates.

Even when autoinhibited, Parkin exists in an equilibrium involving rapid intra-molecular movements [89]. Such domain flexibility often hampers successful crystallisation efforts. Not surprisingly, therefore, crystallisation of pParkin ${ }_{\triangle R E P \triangle R c a t}: \mathrm{pUB}$ and $\mathrm{p} B d$ Parkin:pUB:UBE2L3, required some noteworthy adjustments. In the case of pParkin $\triangle R E P \triangle R c a t:$ pUB, the complex was trapped only with deletion of REP and Rcat domain. In the case of pBdParkin:pUB:UBE2L3, crystallisation of the complex was facilitated by crosslinking the C-terminus of UBE2L3 to the $\mathrm{N}$-terminus of Parkin UBL domain via a ten-residue linker. This linker is not visible in the crystal structure; thus, it is not possible to work out whether the UBE2L3-Parkin interaction or pUBL-RING0 interaction occurs in trans or cis. While the authors have deposited the crystal structure as a single, fusion polypeptide, they hint that it is more likely that UBE2L3 binds to Parkin RING1 in cis, while the pUBL, displaced as a result of either this interaction, or because of the contortion caused by the crosslinking, then interacts in trans with RING0 of a neighbouring fusion polypeptide. Nevertheless, the latter structure validates the former, as in spite of inclusion of REP and Rcat domains in the fusion polypeptide, these regions are not visible in the $\mathrm{pBdParkin}$ :pUB:UBE2L3 model, suggesting that pUBL-RING0 interaction indeed releases Rcat, and that in the absence of the donor UB, Rcat is destabilised. Also, consistent with the domain movements shown in these crystal structures relative to those of autoinhibited Parkin, hydrogen-deuterium exchange mass spectrometry (HDX-MS) data from three independent reports confirm that: (1) pUB binding increases UBL solvent-exposure; (2) phosphorylation of UBL leads to re-stabilisation of UBL and reduced solvent-exposure; and (3) concomitant with this, Rcat is released and exposed to solvent $[65,68,83]$.

\subsection{Cis, trans or the whole spectrum}

Importantly, both the cis- and trans-activating models are based on crystallographic models where the allosteric pUB is monomeric, as is the case with the in vitro assays set out to test these hypothetical models (Fig. 6A). In cellular environment, however, the OMM of damaged mitochondria displays UB chains predominantly with Lys6, Lys11 and Lys63 linkage topologies, where the Ser65-phosphorylation status of the UB chains is sub-stoichiometric [24,80]. Thus, Parkin is likely to be recruited on pUB chains, where the pUB is linked to other (p)UB moieties on its distal and/or proximal sites. Inspection of pUB in activated Parkin structures reveal that Lys6 and Lys11 in particular protrude towards the cryptic donor UB binding site $[68,69]$. This raises the possibility that while a pUB moiety recruits Parkin and triggers the allosteric activation mechanism as described above, a neighbouring (p)UB moiety may occlude recruitment of donor UB, thus preventing that Parkin molecule from forming a fruitful complex with an E2 UB conjugate (Fig. 6B). In line with this, in vitro self-ubiquitylation experiments involving Lys6-linked di-pUB, where either distal, proximal or both moieties are phosphorylated (hereafter pUB-UB, UB-pUB and pUB-pUB, respectively) depict complex outcomes. Interestingly, both pUB-pUB and pUB-UB activate Parkin, while UB-pUB fails to do so, with this shortcoming relieved by UBL phosphorylation [91]. Thus, although the cis- and trans-activation models have often been discussed as being mutually exclusive [83,92], in reality Parkin's cellular environment may necessitate elements from both cis- and trans-activating models, such as pUBL binding to RING0 to release Rcat, as illustrated by the recent structures, coupled to the ability of two Parkin molecules to dimerise around one E2 $\sim$ UB to mediate UB-transfer to Rcat, similar to that observed for HOIP.
A

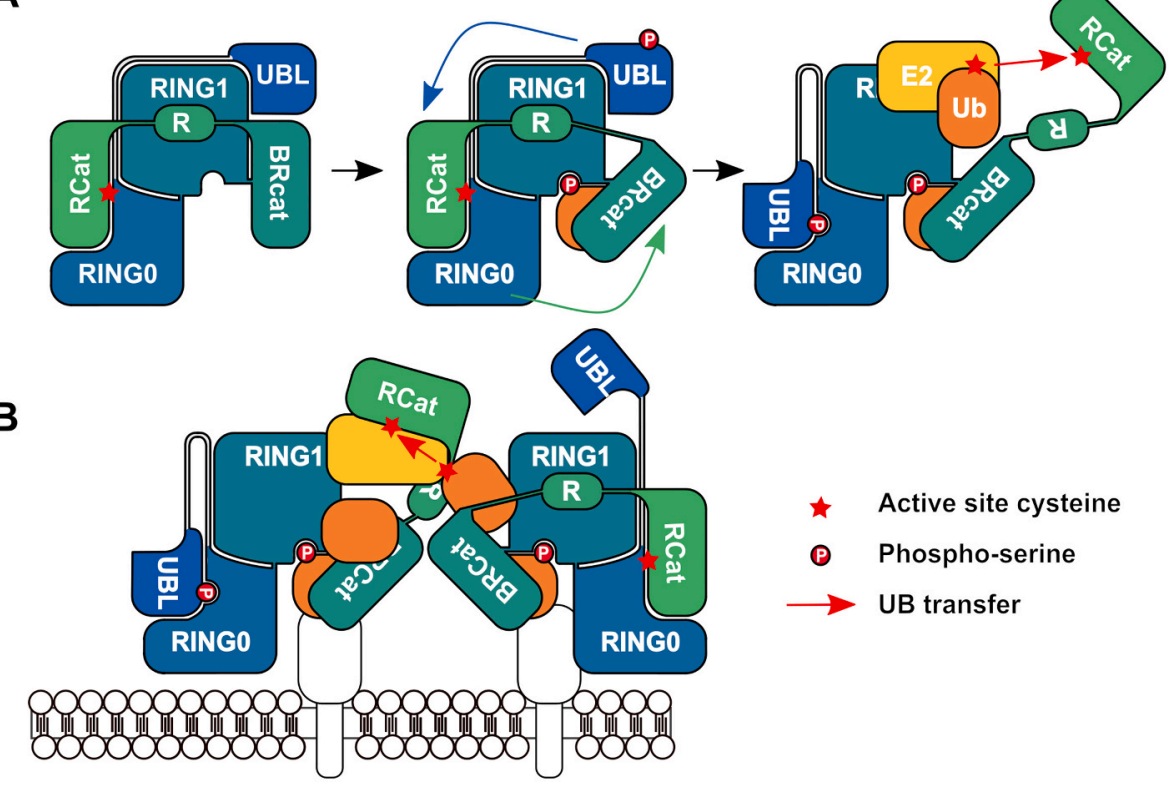

Fig. 6. cis- $v s$ trans-activation models explaining mechanism of Parkin activation.

(A) Box-diagrams showing how pUB-binding and phosphorylation of the UBL domain push a single molecule of Parkin into the fully activated state. Parkin is coloured as in Fig. 1C, while E2 and UB are coloured yellow and orange, respectively. Blue-green arrows indicate domain movements triggered by (p) UB binding. (B) Box-diagrams showing how multiple molecules of Parkin may be forced to dimerise around a E2 UB conjugate in order to overcome the steric hindrance caused by poly-pUB chains on OMM proteins of damaged mitochondria. Parkin, E2 and UB and are coloured as in panel A, while OMM phospholipid bi-layer and integral membrane proteins are coloured as while circles. OMM integral membrane proteins are shown as white boxes, with pUB moieties attached on them coloured orange. 


\section{Concluding remarks}

Harbouring over 130 distinct mutations observed in over 1000 patients with early-onset PD, Parkin has been the hot-spot of signalling pathways involved in PD aetiology. Extensive cell-based studies revealed that Parkin functions under PINK1 and generates a feedforward signalling mechanism that mediates mitophagy. Over the past two decades the question of how Parkin activity is regulated has been extensively studied, demonstrating that: (1) inter-domain interactions exert a tight inhibition on Parkin activity; (2) Parkin-pUB interaction relieves this auto-inhibition; and (3) phosphorylation of Parkin UBL shifts the equilibrium towards maximal Parkin activation. Specifically, data obtained using molecules in solution depict clearly that following pUB binding and phosphorylation of the UBL domain, Parkin can unravel, allowing the Rcat domain to traverse across the central RING1 domain to meet the E2 UB conjugate. While a large body of work elucidates how Parkin behaves in solution, molecular detail of how Parkin activation proceeds in its cellular context remains elusive. It remains to be determined whether, as some evidence suggests, in such a complex environment Parkin molecules dimerise around one $\mathrm{E} 2 \sim \mathrm{UB}$ to mediate UB-transfer to Rcat. Furthermore, little information exists on how activated Parkin can then modify such a wide variety of substrates. The next decade, therefore, is likely to reveal how these rearrangements and modes of activation support Parkin substrate targeting.

\section{Credit author statement}

MG, RT and GS wrote the manuscript with input from HW and GSS.

\section{Declaration of Competing Interest}

Authors declare no conflict of interest.

\section{Acknowledgements}

GSS was funded by Canadian Institutes of Health Research (CIHR) PJT-166019. HW was funded by Wellcome Trust Investigator Award $(209347 / \mathrm{Z} / 17 / \mathrm{Z})$

\section{References}

[1] O.B. Tysnes, A. Storstein, Epidemiology of Parkinson's disease, J. Neural Transm. 124 (2017) 901-905, https://doi.org/10.1007/s00702-017-1686-y.

[2] L.L.M. de Lau, M.M.B. Breteler, Epidemiology of Parkinson's disease, Lancet Neurol. 5 (2006) 525-535.

[3] A.J. Lees, The Parkinson chimera, Neurology 72 (2009), https://doi.org/10.1212/ WNL.0b013e318198daec.

[4] S. Miller, M.M.K. Muqit, Therapeutic approaches to enhance PINK1/Parkin mediated mitophagy for the treatment of Parkinson's disease, Neurosci. Lett. 705 (2019) 7-13, https://doi.org/10.1016/j.neulet.2019.04.029.

[5] J. William Langston, P. Ballard, J.W. Tetrud, I. Irwin, Chronic parkinsonism in humans due to a product of meperidine-analog synthesis, Science. 219 (1983) 979-980, https://doi.org/10.1126/science.6823561.

[6] X. Reed, S. Bandrés-Ciga, C. Blauwendraat, M.R. Cookson, The role of monogenic genes in idiopathic Parkinson's disease, Neurobiol. Dis. 124 (2019) 230-239, https://doi.org/10.1016/j.nbd.2018.11.012.

[7] M. Borsche, S.L. Pereira, C. Klein, A. Grünewald, Mitochondria and Parkinson's disease: clinical, molecular, and translational aspects, J. Parkinsons Dis. (2020) 1-16, https://doi.org/10.3233/jpd-201981.

[8] E.M. Valente, A.R. Bentivoglio, P.H. Dixon, A. Ferraris, T. Lalongo, M. Frontali, A. Albanese, N.W. Wood, Localization of a novel locus for autosomal recessive early-onset parkinsonism, PARK6, on human chromosome 1p35-p36, Am. J. Hum. Genet. 68 (2001) 895-900, https://doi.org/10.1086/319522.

[9] E.M. Valente, P.M. Abou-Sleiman, V. Caputo, M.M.K. Muqit, K. Harvey, S. Gispert, Z. Ali, D. Del Turco, A.R. Bentivoglio, D.G. Healy, A. Albanese, R. Nussbaum, R. González-Maldonado, T. Deller, S. Salvi, P. Cortelli, W.P. Gilks, D.S. Latchman, R.J. Harvey, B. Dallapiccola, G. Auburger, N.W. Wood, Hereditary early-onset Parkinson's disease caused by mutations in PINK1, Science. 304 (2004) 1158-1160, https://doi.org/10.1126/science.1096284.

[10] T. Kitada, S. Asakawa, N. Hattori, H. Matsumine, Y. Yamamura, S. Minoshima, M. Yokochi, Y. Mizuno, N. Shimizu, Mutations in the parkin gene cause autosomal recessive juvenile parkinsonism, Nature. 392 (1998) 605-608, https://doi.org/ $10.1038 / 33416$.
[11] M. Kasten, C. Hartmann, J. Hampf, S. Schaake, A. Westenberger, E.J. Vollstedt, A. Balck, A. Domingo, F. Vulinovic, M. Dulovic, I. Zorn, H. Madoev, H. Zehnle, C. M. Lembeck, L. Schawe, J. Reginold, J. Huang, I.R. König, L. Bertram, C. Marras, K. Lohmann, C.M. Lill, C. Klein, Genotype-phenotype relations for the Parkinson's disease genes Parkin, PINK1, DJ1: MDSGene systematic review, Mov. Disord. 33 (2018) 730-741, https://doi.org/10.1002/mds.27352.

[12] M.M.X. Tan, N. Malek, M.A. Lawton, L. Hubbard, A.M. Pittman, T. Joseph, J. Hehir, D.M.A. Swallow, K.A. Grosset, S.L. Marrinan, N. Bajaj, R.A. Barker, D. J. Burn, C. Bresner, T. Foltynie, J. Hardy, N. Wood, Y. Ben-Shlomo, D.G. Grosset, N. M. Williams, H.R. Morris, Genetic analysis of Mendelian mutations in a large UK population-based Parkinson's disease study, Brain. 142 (2019) 2828-2844, https://doi.org/10.1093/brain/awz191.

[13] S. Taghavi, R. Chaouni, A. Tafakhori, L.J. Azcona, S.G. Firouzabadi, M.D. Omrani, J. Jamshidi, B. Emamalizadeh, G.A. Shahidi, M. Ahmadi, S.A.H. Habibi, A. Ahmadifard, A. Fazeli, M. Motallebi, P. Petramfar, S. Askarpour, S. Askarpour, H.A. Shahmohammadibeni, N. Shahmohammadibeni, H. Eftekhari, A.E. Shafiei Zarneh, S. Mohammadihosseinabad, M. Khorrami, S. Najmi, A. Chitsaz, P. Shokraeian, H. Ehsanbakhsh, J. Rezaeidian, R. Ebrahimi Rad, F. Madadi, M. Andarva, E. Alehabib, M. Atakhorrami, S.E. Mortazavi, Z. Azimzadeh, M. Bayat, A.M. Besharati, M.A. Harati-Ghavi, S. Omidvari, Z. Dehghani-Tafti, F. Mohammadi, B. Mohammad Hossein Pour, H. Noorollahi Moghaddam, E. Esmaili Shandiz, A. Habibi, Z. Taherian-Esfahani, H. Darvish, C. Paisán-Ruiz, A clinical and molecular genetic study of 50 families with autosomal recessive parkinsonism revealed known and novel gene mutations, Mol. Neurobiol. 55 (2018) 3477-3489, https://doi.org/10.1007/s12035-017-0535-1.

[14] S.M. Jin, M. Lazarou, C. Wang, L.A. Kane, D.P. Narendra, R.J. Youle, Mitochondrial membrane potential regulates PINK1 import and proteolytic destabilization by PARL, J. Cell Biol. 191 (2010) 933-942, https://doi.org/10.1083/jcb.201008084.

[15] E. Deas, H. Plun-Favreau, S. Gandhi, H. Desmond, S. Kjaer, S.H.Y. Loh, A.E. M. Renton, R.J. Harvey, A.J. Whitworth, L.M. Martins, A.Y. Abramov, N.W. Wood, PINK1 cleavage at position A103 by the mitochondrial protease PARL, Hum. Mol. Genet. 20 (2011) 867-879, https://doi.org/10.1093/hmg/ddq526.

[16] L.A. Kane, M. Lazarou, A.I. Fogel, Y. Li, K. Yamano, S.A. Sarraf, S. Banerjee, R. J. Youle, PINK1 phosphorylates ubiquitin to activate Parkin E3 ubiquitin ligase activity, J. Cell Biol. 205 (2014) 143-153, https://doi.org/10.1083/ jcb. 201402104.

[17] A. Kazlauskaite, C. Kondapalli, R. Gourlay, D.G. Campbell, M.S. Ritorto, K. Hofmann, D.R. Alessi, A. Knebel, M. Trost, M.M.K. Muqit, Accelerated publication: Parkin is activated by PINK1-dependent phosphorylation of ubiquitin at Ser65, Biochem. J. 460 (2014) 127-139, https://doi.org/10.1042/BJ20140334.

[18] F. Koyano, K. Okatsu, H. Kosako, Y. Tamura, E. Go, M. Kimura, Y. Kimura, H. Tsuchiya, H. Yoshihara, T. Hirokawa, T. Endo, E.A. Fon, J.F. Trempe, Y. Saeki, K. Tanaka, N. Matsuda, Ubiquitin is phosphorylated by PINK1 to activate parkin, Nature. 510 (2014) 162-166, https://doi.org/10.1038/nature13392.

[19] D. Narendra, A. Tanaka, D.-F. Suen, R.J. Youle, Parkin is recruited selectively to impaired mitochondria and promotes their autophagy, J. Cell Biol. 183 (2008) 795-803, https://doi.org/10.1083/jcb.200809125.

[20] C. Kondapalli, A. Kazlauskaite, N. Zhang, H.I. Woodroof, D.G. Campbell, R. Gourlay, L. Burchell, H. Walden, T.J. Macartney, M. Deak, A. Knebel, D. R. Alessi, M.M.K. Muqit, PINK1 is activated by mitochondrial membrane potential depolarization and stimulates Parkin E3 ligase activity by phosphorylating serine 65, Open Biol. 2 (2012) 120080, https://doi.org/10.1098/rsob.120080.

[21] K. Shiba-Fukushima, Y. Imai, S. Yoshida, Y. Ishihama, T. Kanao, S. Sato, N. Hattori, PINK1-mediated phosphorylation of the Parkin ubiquitin-like domain primes mitochondrial translocation of Parkin and regulates mitophagy, Sci. Rep. 2 (2012) 1-8, https://doi.org/10.1038/srep01002.

[22] N. Matsuda, S. Sato, K. Shiba, K. Okatsu, K. Saisho, C.A. Gautier, Y. Sou, S. Saiki, S. Kawajiri, F. Sato, M. Kimura, M. Komatsu, N. Hattori, K. Tanaka, PINK1 stabilized by mitochondrial depolarization recruits Parkin to damaged mitochondria and activates latent Parkin for mitophagy, J. Cell Biol. 189 (2010) 211-221, https://doi.org/10.1083/jcb.200910140.

[23] A. Kazlauskaite, C. Kondapalli, R. Gourlay, D.G. Campbell, M.S. Ritorto, K. Hofmann, D.R. Alessi, A. Knebel, M. Trost, M.M.K. Muqit, Accelerated publication: Parkin is activated by PINK1-dependent phosphorylation of ubiquitin at Ser65, Biochem. J. 460 (2014) 127-139, https://doi.org/10.1042/BJ20140334.

[24] A. Ordureau, J.A. Paulo, W. Zhang, T. Ahfeldt, J. Zhang, E.F. Cohn, Z. Hou, J.M. Heo, L.L. Rubin, S.S. Sidhu, S.P. Gygi, J.W. Harper, Dynamics of PARKINdependent mitochondrial ubiquitylation in induced neurons and model systems revealed by digital snapshot proteomics, Mol. Cell 70 (2018) 211-227, e8, https ://doi.org/10.1016/j.molcel.2018.03.012.

[25] A. Plechanovová, E.G. Jaffray, M.H. Tatham, J.H. Naismith, R.T. Hay, Structure of a RING E3 ligase and ubiquitin-loaded E2 primed for catalysis, Nature. 489 (2012) 115-120, https://doi.org/10.1038/nature11376.

[26] J.N. Pruneda, P.J. Littlefield, S.E. Soss, K.A. Nordquist, W.J. Chazin, P.S. Brzovic, R.E. Klevit, Structure of an E3:E2 Ub complex reveals an allosteric mechanism shared among RING/U-box ligases, Mol. Cell 47 (2012) 933-942, https://doi.org/ 10.1016/j.molcel.2012.07.001.

[27] L.E. Fritsch, M.E. Moore, S.A. Sarraf, A.M. Pickrell, Ubiquitin and receptordependent Mitophagy pathways and their implication in neurodegeneration, J. Mol. Biol. (2019), https://doi.org/10.1016/j.jmb.2019.10.015.

[28] K. Tanaka, The PINK1-parkin axis: an overview, Neurosci. Res. (2020), https://doi. org/10.1016/j.neures.2020.01.006.

[29] A.M. Pickrell, R.J. Youle, The roles of PINK1, Parkin, and mitochondrial fidelity in Parkinson's disease, Neuron. 85 (2015) 257-273, https://doi.org/10.1016/j. neuron.2014.12.007. 
[30] W. Li, M.H. Bengtson, A. Ulbrich, A. Matsuda, V.A. Reddy, A. Orth, S.K. Chanda, S. Batalov, C.A.P. Joazeiro, Genome-wide and functional annotation of human E3 ubiquitin ligases identifies MULAN, a mitochondrial E3 that regulates the Organelle's dynamics and signaling, PLoS One 3 (2008), e1487, https://doi.org/ 10.1371/journal.pone.0001487.

[31] A.L. Haas, I.A. Rose, The mechanism of ubiquitin activating enzyme. A kinetic and equilibrium analysis, J. Biol. Chem. 257 (1982) 10329-10337.

[32] A.L. Haas, J.V. Warms, A. Hershko, I.A. Rose, Ubiquitin-activating enzyme. Mechanism and role in protein-ubiquitin conjugation, J. Biol. Chem. 257 (1982) 2543-2548.

[33] M.D. Stewart, T. Ritterhoff, R.E. Klevit, P.S. Brzovic, E2 enzymes: more than just middle men, Cell Res. 26 (2016) 423-440, https://doi.org/10.1038/cr.2016.35.

[34] D. Komander, M. Rape, The ubiquitin code, Annu. Rev. Biochem. 81 (2012) 203-229, https://doi.org/10.1146/annurev-biochem-060310-170328.

[35] O. Kerscher, R. Felberbaum, M. Hochstrasser, Modification of proteins by ubiquitin and ubiquitin-like proteins, Annu. Rev. Cell Dev. Biol. 22 (2006) 159-180, https:// doi.org/10.1146/annurev.cellbio.22.010605.093503.

[36] C. Hoege, B. Pfander, G.-L. Moldovan, G. Pyrowolakis, S. Jentsch, RAD6-dependent DNA repair is linked to modification of PCNA by ubiquitin and SUMO, Nature. 419 (2002) 135-141, https://doi.org/10.1038/nature00991.

[37] S. Ghosh, T. Saha, Central role of Ubiquitylation in genome maintenance: DNA replication and damage repair, ISRN Mol. Biol. 2012 (2012) 146748, https://doi org /10.5402/2012/146748.

[38] H. Walden, A.J. Deans, The Fanconi anemia DNA repair pathway: structural and functional insights into a complex disorder, Annu. Rev. Biophys. 43 (2014) 257-278, https://doi.org/10.1146/annurev-biophys-051013-022737.

[39] M.L. Rennie, K. Lemonidis, C. Arkinson, V.K. Chaugule, M. Clarke, J. Streetley, L. Spagnolo, H. Walden, Differential functions of FANCI and FANCD2 ubiquitylation stabilize ID2 complex on DNA, EMBO Rep. 21 (2020), e50133, https://doi.org/10.15252/embr.202050133.

[40] A. Ciechanover, P. Brundin, The ubiquitin proteasome system in neurodegenerative diseases: sometimes the chicken, sometimes the egg, Neuron. 40 (2003) 427-446, https://doi.org/10.1016/S0896-6273(03)00606-8.

[41] C. Uchida, M. Kitagawa, RING-, HECT-, and RBR-type E3 ubiquitin ligases: involvement in human cancer, Curr. Cancer Drug Targets 16 (2016) 157-174, https://doi.org/10.2174/1568009616666151112122801.

[42] L. Buetow, D.T. Huang, Structural insights into the catalysis and regulation of E3 ubiquitin ligases, Nat. Rev. Mol. Cell Biol. 17 (2016) 626-642, https://doi.org/ 10.1038/nrm.2016.91.

[43] R.J. Deshaies, C.A.P. Joazeiro, RING Domain E3 Ubiquitin Ligases, Annu. Rev. Biochem. 78 (2009) 399-434, https://doi.org/10.1146/annurev. biochem.78.101807.093809.

[44] B.E. Riley, J.C. Lougheed, K. Callaway, M. Velasquez, E. Brecht, L. Nguyen, T. Shaler, D. Walker, Y. Yang, K. Regnstrom, L. Diep, Z. Zhang, S. Chiou, M. Bova, D.R. Artis, N. Yao, J. Baker, T. Yednock, J.A. Johnston, Structure and function of Parkin E3 ubiquitin ligase reveals aspects of RING and HECT ligases, Nat. Commun. 4 (2013) 1982, https://doi.org/10.1038/ncomms2982.

[45] F. Bernassola, M. Karin, A. Ciechanover, G. Melino, The HECT family of E3 ubiquitin ligases: multiple players in cancer development, Cancer Cell 14 (2008) 10-21, https://doi.org/10.1016/j.ccr.2008.06.001.

[46] M.B. Metzger, V.A. Hristova, A.M. Weissman, HECT and RING finger families of E3 ubiquitin ligases at a glance, J. Cell Sci. 125 (2012) 531-537, https://doi.org/ 10.1242/jcs.091777.

[47] D. Rotin, S. Kumar, Physiological functions of the HECT family of ubiquitin ligases, Nat. Rev. Mol. Cell Biol. 10 (2009) 398-409, https://doi.org/10.1038/nrm2690.

[48] H. Walden, K. Rittinger, RBR ligase-mediated ubiquitin transfer: a tale with many twists and turns, Nat. Struct. Mol. Biol. 25 (2018) 440-445, https://doi.org/ 10.1038/s41594-018-0063-3.

[49] K.K. Dove, R.E. Klevit, RING-between-RING E3 ligases: emerging themes amid the variations, J. Mol. Biol. 429 (2017) 3363-3375, https://doi.org/10.1016/j. jmb.2017.08.008.

[50] D.M. Wenzel, A. Lissounov, P.S. Brzovic, R.E. Klevit, UBCH7 reactivity profile reveals parkin and HHARI to be RING/HECT hybrids, Nature. 474 (2011) 105-108, https://doi.org/10.1038/nature09966.

[51] D.E. Spratt, R. Julio Martinez-Torres, Y.J. Noh, P. Mercier, N. Manczyk, K. R. Barber, J.D. Aguirre, L. Burchell, A. Purkiss, H. Walden, G.S. Shaw, A molecular explanation for the recessive nature of parkin-linked Parkinson's disease, Nat. Commun. 4 (2013) 1983, https://doi.org/10.1038/ncomms2983.

[52] S.A. Beasley, V.A. Hristova, G.S. Shaw, Structure of the Parkin in-between-ring domain provides insights for E3-ligase dysfunction in autosomal recessive Parkinson's disease, Proc. Natl. Acad. Sci. 104 (2007) 3095-3100, https://doi.org/ 10.1073/pnas.0610548104.

[53] D.E. Spratt, H. Walden, G.S. Shaw, RBR E3 ubiquitin ligases: new structures, new insights, new questions, Biochem. J. 458 (2014) 421-437, https://doi.org/ 10.1042/BJ20140006.

[54] K.S. Hamilton, M.J. Ellison, K.R. Barber, R.S. Williams, J.T. Huzil, S. McKenna, C. Ptak, M. Glover, G.S. Shaw, Structure of a conjugating enzyme-ubiquitin Thiolester intermediate reveals a novel role for the ubiquitin tail, Structure. 9 (2001) 897-904, https://doi.org/10.1016/S0969-2126(01)00657-8.

[55] J.N. Pruneda, K.E. Stoll, L.J. Bolton, P.S. Brzovic, R.E. Klevit, Ubiquitin in motion: structural studies of the ubiquitin-conjugating enzyme $\sim$ ubiquitin conjugate, Biochemistry. 50 (2011) 1624-1633, https://doi.org/10.1021/bi101913m.

[56] A. Saha, S. Lewis, G. Kleiger, B. Kuhlman, R.J. Deshaies, Essential role for ubiquitin-ubiquitin-conjugating enzyme interaction in ubiquitin discharge from Cdc34 to substrate, Mol. Cell 42 (2011) 75-83, https://doi.org/10.1016/j. molcel.2011.03.016.
[57] J.N. Pruneda, P.J. Littlefield, S.E. Soss, K.A. Nordquist, W.J. Chazin, P.S. Brzovic, R.E. Klevit, Structure of an E3:E2 Ub complex reveals an allosteric mechanism shared among RING/U-box ligases, Mol. Cell 47 (2012) 933-942, https://doi.org/ 10.1016/j.molcel.2012.07.001.

[58] K.K. Dove, J.L. Olszewski, L. Martino, D.M. Duda, X.S. Wu, D.J. Miller, K.H. Reiter, K. Rittinger, B.A. Schulman, R.E. Klevit, Structural studies of HHARI/UbcH7 Ub reveal unique E2 $\sim$ Ub conformational restriction by RBR RING1, Structure 25 (2017) 890-900, e5, https://doi.org/10.1016/j.str.2017.04.013.

[59] K.S. Hamilton, M.J. Ellison, K.R. Barber, R.S. Williams, J.T. Huzil, S. McKenna, C. Ptak, M. Glover, G.S. Shaw, Structure of a conjugating enzyme-ubiquitin Thiolester intermediate reveals a novel role for the ubiquitin tail, Structure. 9 (2001) 897-904, https://doi.org/10.1016/S0969-2126(01)00657-8.

[60] H. Dou, L. Buetow, G.J. Sibbet, K. Cameron, D.T. Huang, BIRC7-E2 ubiquitin conjugate structure reveals the mechanism of ubiquitin transfer by a RING dimer, Nat. Struct. Mol. Biol. 19 (2012) 876-883, https://doi.org/10.1038/nsmb.2379.

[61] J. Song, J. Wang, A.A. Jozwiak, W. Hu, P.M. Swiderski, Y. Chen, Stability of thioester intermediates in ubiquitin-like modifications: stability of thioester intermediates, Protein Sci. 18 (2009) 2492-2499, https://doi.org/10.1002/ pro.254.

[62] T. Wauer, D. Komander, Structure of the human Parkin ligase domain in an autoinhibited state, EMBO J. 32 (2013) 2099-2112, https://doi.org/10.1038/ emboj.2013.125.

[63] K.K. Dove, J.L. Olszewski, L. Martino, D.M. Duda, X.S. Wu, D.J. Miller, K.H. Reiter, K. Rittinger, B.A. Schulman, R.E. Klevit, Structural studies of HHARI/UbcH7 Ub reveal unique E2 $\sim$ Ub conformational restriction by RBR RING1, Structure 25 (2017) 890-900, e5, https://doi.org/10.1016/j.str.2017.04.013.

[64] B.C. Lechtenberg, A. Rajput, R. Sanishvili, M.K. Dobaczewska, C.F. Ware, P. D. Mace, S.J. Riedl, Structure of a HOIP/E2 ubiquitin complex reveals RBR E3 ligase mechanism and regulation, Nature. 529 (2016) 546-550, https://doi.org/ 10.1038/nature16511.

[65] T.E. Condos, K.M. Dunkerley, E.A. Freeman, K.R. Barber, J.D. Aguirre, V. K. Chaugule, Y. Xiao, L. Konermann, H. Walden, G.S. Shaw, Synergistic recruitment of UbcH7 Ub and phosphorylated Ubl domain triggers parkin activation, EMBO J. 37 (2018), https://doi.org/10.15252/embj.2018100014.

[67] M. Gundogdu, H. Walden, Structural basis of generic versus specific E2-RING E3 interactions in protein ubiquitylation, Protein Sci. 28 (2019) 1758-1770, https:// doi.org/10.1002/pro.3690.

[68] C. Gladkova, S.L. Maslen, J.M. Skehel, D. Komander, Mechanism of parkin activation by PINK1, Nature. 559 (2018) 410-414, https://doi.org/10.1038/ s41586-018-0224-X.

[69] A. Kumar, V.K. Chaugule, T.E.C. Condos, K.R. Barber, C. Johnson, R. Toth, R. Sundaramoorthy, A. Knebel, G.S. Shaw, H. Walden, Parkin-phosphoubiquitin complex reveals cryptic ubiquitin-binding site required for RBR ligase activity, Nat. Struct. Mol. Biol. 24 (2017) 475-483, https://doi.org/10.1038/nsmb.3400.

[70] S.A. Beasley, V.A. Hristova, G.S. Shaw, Structure of the Parkin in-between-ring domain provides insights for E3-ligase dysfunction in autosomal recessive Parkinson's disease, Proc. Natl. Acad. Sci. 104 (2007) 3095-3100, https://doi.org/ 10.1073/pnas.0610548104.

[71] D.E. Spratt, R. Julio Martinez-Torres, Y.J. Noh, P. Mercier, N. Manczyk, K. R. Barber, J.D. Aguirre, L. Burchell, A. Purkiss, H. Walden, G.S. Shaw, A molecular explanation for the recessive nature of parkin-linked Parkinson's disease, Nat. Commun. 4 (2013) 1983, https://doi.org/10.1038/ncomms2983.

[72] J.-F. Trempe, V. Sauve, K. Grenier, M. Seirafi, M.Y. Tang, M. Menade, S. Al-AbdulWahid, J. Krett, K. Wong, G. Kozlov, B. Nagar, E.A. Fon, K. Gehring, Structure of Parkin reveals mechanisms for ubiquitin ligase activation, Science. 340 (2013) 1451-1455, https://doi.org/10.1126/science.1237908.

[73] A. Kumar, J.D. Aguirre, T.E. Condos, R.J. Martinez-Torres, V.K. Chaugule, R. Toth, R. Sundaramoorthy, P. Mercier, A. Knebel, D.E. Spratt, K.R. Barber, G.S. Shaw, H. Walden, Disruption of the autoinhibited state primes the E3 ligase parkin for activation and catalysis, EMBO J. 34 (2015) 2506-2521, https://doi.org/ 10.15252/embj.201592337.

[74] Y. Zhang, J. Gao, K.K.K. Chung, H. Huang, V.L. Dawson, T.M. Dawson, Parkin functions as an E2-dependent ubiquitin- protein ligase and promotes the degradation of the synaptic vesicle-associated protein, CDCrel-1, Proc. Natl. Acad. Sci. 97 (2000) 13354-13359, https://doi.org/10.1073/pnas. 240347797.

[75] H. Shimura, N. Hattori, S. Kubo, Y. Mizuno, S. Asakawa, S. Minoshima, N. Shimizu, K. Iwai, T. Chiba, K. Tanaka, T. Suzuki, Familial Parkinson disease gene product, parkin, is a ubiquitin-protein ligase, Nat. Genet. 25 (2000) 302-305, https://doi. org $/ 10.1038 / 77060$.

[76] N. Matsuda, T. Kitami, T. Suzuki, Y. Mizuno, N. Hattori, K. Tanaka, Diverse effects of pathogenic mutations of Parkin that catalyze multiple monoubiquitylation in vitro, J. Biol. Chem. 281 (2006) 3204-3209, https://doi.org/10.1074/jbc. M510393200.

[77] V.K. Chaugule, L. Burchell, K.R. Barber, A. Sidhu, S.J. Leslie, G.S. Shaw, H. Walden, Autoregulation of Parkin activity through its ubiquitin-like domain, EMBO J. 30 (2011) 2853-2867, https://doi.org/10.1038/emboj.2011.204.

[78] M.Y. Tang, M. Vranas, A.I. Krahn, S. Pundlik, J.F. Trempe, E.A. Fon, Structureguided mutagenesis reveals a hierarchical mechanism of Parkin activation, Nat. Commun. 8 (2017), https://doi.org/10.1038/ncomms14697.

[79] L.A. Kane, M. Lazarou, A.I. Fogel, Y. Li, K. Yamano, S.A. Sarraf, S. Banerjee, R. J. Youle, PINK1 phosphorylates ubiquitin to activate parkin E3 ubiquitin ligase activity, J. Cell Biol. 205 (2014) 143-153, https://doi.org/10.1083/ jcb. 201402104.

[80] A. Ordureau, J.M. Heo, D.M. Duda, J.A. Paulo, J.L. Olszewski, D. Yanishevski, J. Rinehart, B.A. Schulman, J.W. Harper, Defining roles of PARKIN and ubiquitin phosphorylation by PINK1 in mitochondrial quality control using a ubiquitin 
replacement strategy, Proc. Natl. Acad. Sci. U. S. A. 112 (2015) 6637-6642, https://doi.org/10.1073/pnas.1506593112.

[81] V. Sauvé, A. Lilov, M. Seirafi, M. Vranas, S. Rasool, G. Kozlov, T. Sprules, J. Wang, J. Trempe, K. Gehring, A Ubl/ubiquitin switch in the activation of Parkin, EMBO J. 34 (2015) 2492-2505, https://doi.org/10.15252/embj.201592237.

[82] T. Wauer, M. Simicek, A. Schubert, D. Komander, Mechanism of phosphoubiquitin-induced PARKIN activation, Nature. 524 (2015) 370-374, https://doi. org/10.1038/nature14879.

[83] V. Sauvé, G. Sung, N. Soya, G. Kozlov, N. Blaimschein, L.S. Miotto, J.F. Trempe, G. L. Lukacs, K. Gehring, Mechanism of parkin activation by phosphorylation, Nat. Struct. Mol. Biol. 25 (2018) 623-630, https://doi.org/10.1038/s41594-018-00887.

[84] V. Sauvé, G. Sung, N. Soya, G. Kozlov, N. Blaimschein, L.S. Miotto, J.F. Trempe, G. L. Lukacs, K. Gehring, Mechanism of parkin activation by phosphorylation, Nat. Struct. Mol. Biol. 25 (2018) 623-630, https://doi.org/10.1038/s41594-018-0088 7.

[85] M. Gundogdu, H. Walden, Structural basis of generic versus specific E2-RING E3 interactions in protein ubiquitylation, Protein Sci. 28 (2019) 1758-1770, https:// doi.org/10.1002/pro.3690.

[86] B.C. Lechtenberg, A. Rajput, R. Sanishvili, M.K. Dobaczewska, C.F. Ware, P. D. Mace, S.J. Riedl, Structure of a HOIP/E2 ubiquitin complex reveals RBR E3 ligase mechanism and regulation, Nature. 529 (2016) 546-550, https://doi.org/ 10.1038/nature16511.

[87] X. Zheng, T. Hunter, Parkin mitochondrial translocation is achieved through a novel catalytic activity coupled mechanism, Cell Res. 23 (2013) 886-897, https:// doi.org/10.1038/cr.2013.66.
[88] M. Lazarou, D.P. Narendra, S.M. Jin, E. Tekle, S. Banerjee, R.J. Youle, PINK1 drives Parkin self-association and HECT-like E3 activity upstream of mitochondrial binding, J. Cell Biol. 200 (2013) 163-172, https://doi.org/10.1083/ jcb. 201210111.

[89] T.R. Caulfield, F.C. Fiesel, E.L. Moussaud-Lamodière, D.F.A.R. Dourado, S. C. Flores, W. Springer, Phosphorylation by PINK1 Releases the UBL domain and initializes the conformational opening of the E3 Ubiquitin Ligase Parkin, PLoS Comput. Biol. 10 (2014), https://doi.org/10.1371/journal.pcbi.1003935.

[90] J.D. Aguirre, K.M. Dunkerley, P. Mercier, G.S. Shaw, Structure of phosphorylated UBL domain and insights into PINK1-orchestrated parkin activation, PNAS (2016), https://doi.org/10.1073/pnas.1613040114.

[91] M. Pan, Q. Zheng, S. Gao, Q. Qu, Y. Yu, M. Wu, H. Lan, Y. Li, S. Liu, J. Li, D. Sun, L. Lu, T. Wang, W. Zhang, J. Wang, Y. Li, H.-G. Hu, C. Tian, L. Liu, Chemical synthesis of structurally defined phosphorylated ubiquitins suggests impaired parkin activation by phosphorylated ubiquitins with a non-phosphorylated distal unit, CCS Chem. 1 (2019) 476-489, https://doi.org/10.31635/ ccschem.019.20190001.

[92] A.N. Bayne, J.-F. Trempe, Mechanisms of PINK1, ubiquitin and Parkin interactions in mitochondrial quality control and beyond, Cell. Mol. Life Sci. 76 (2019) 4589-4611, https://doi.org/10.1007/s00018-019-03203-4.

[93] A. Kazlauskaite, R.J. Martínez-Torres, S. Wilkie, A. Kumar, J. Peltier, A. Gonzalez, C. Johnson, J. Zhang, A.G. Hope, M. Peggie, M. Trost, D.M.F. van Aalten, D. R. Alessi, A.R. Prescott, A. Knebel, H. Walden, M.M.K. Muqit, Binding to serine 65phosphorylated ubiquitin primes Parkin for optimal PINK1-dependent phosphorylation and activation, EMBO Rep. 16 (2015) 939-954, https://doi.org/ 10.15252/embr.201540352. 